\title{
PROCEEDINGS OF THE SOCIETY OF BRITISH NEUROLOGICAL SURGEONS: 54th MEETING
}

The 54th meeting of the Society of British Neurological Surgeons was held in Bristol from November 22 to 24, 1956, the President, W. R. Henderson (Leeds), in the chair.

Subdural Hydroma: Problems of Diagnosis

D. G. PHILlips (Bristol) presented a study of 34 cases of subdural hydroma. There was a history of head injury in 22 cases. Problems of diagnosis presented at three stages, clinical, radiological, and surgical.

The clinical syndromes resembled those of subdural haematoma but might be more insidious, particularly in chronic cases. Headache was the commonest symptom but had no characteristic features and was not invariable.

Carotid angiography revealed abnormality in only four out of nine cases. The assessment of the findings with air encephalography was difficult. In cases where subdural fluid was subsequently drained, subdural air was usually present in the first films, but showed in much greater amounts, usually with a fluid level, after 24 or $\mathbf{4 8}$ hours. Results of drainage in these cases were encouraging.

In a comparable number of cases where air encephalography showed subdural air, exploratory burr holes were negative, or no further procedure was undertaken. Practically all of those having burr holes had relief of symptoms, along with half of those having no further procedure. Negative exploratory cranial burr holes might be misleading. It was often noted that fluid, not found with parietal burr holes with the patient supine, was present in the frontal region. The fluid was found in that part of the cranium which was uppermost.

The quantities of subdural fluid actually collected in 22 cases varied from a few millilitres up to $270 \mathrm{ml}$. (average $67 \mathrm{ml}$.). It was suggested that the amounts actually present must have been greatly in excess of these figures. Protein content of the fluid in 27 cases varied from $85 \mathrm{mg}$. $\%$ to $5 \mathrm{~g}$. $\%$ (average $1.3 \mathrm{~g} . \%$ ). In 20 of these cases the content was $500 \mathrm{mg}$. $\%$ or more. These high figures, where there had been recent air encephalography, suggested that the fluid loculation antedated the examination. In these cases lumbar cerebrospinal fluid protein contents were low.

It was not thought that psychological complications played a significant part in this series. Burr holes were not made in a number of cases because of the wish to avoid such complications. A number of these cases might be missed because of the uncertain means of diagnosis.

\section{Acute Intradural Haematoma}

A. Hulme (Bristol) presented a review of 32 cases of acute intradural haematoma. All cases were admitted within 24 hours of receipt of their injury. Although the prognosis was generally bad, a certain number made an excellent recovery following early evacuation of subdural or intracerebral blood clot. Of 32 cases, 15 survived and 10 made an excellent recovery. None of the survivors failed to make a useful recovery.

The most important criterion for early operation was deterioration in the level of consciousness. The importance of deterioration after only partial recovery of consciousness-the "semi-lucid interval"-was emphasized. Only a minority of cases exhibited a clear-cut lucid interval. Eighteen cases showed unilateral or bilateral fixed dilated pupils, and 11 decerebrate posture on admission. The prognosis in cases showing evidence of advanced brain-stem compression was naturally worst, and of patients showing a combination of these signs only three survived. However, even in these cases the outlook was not necessarily hopeless, provided that the situation was dealt with promptly.

Multiple blood clots were found not infrequently. Combined extradural and intradural collections were encountered in seven instances. Operation was usually preceded by bilateral diagnostic burr holes. In some instances arteriography was of great value.

Treatment consisted of evacuation of blood clot, for which a craniotomy was usually required. In the majority of cases solid clot was encountered. Intrathecal injection of saline to reduce tentorial impaction proved valuable in a number of instances. Many of these patients passed through a critical period during the early post-operative phase and skilled nursing played a large part in their survival. The extent to which neurological recovery could occur, often over long periods, was impressive.

\section{The Importance of Shock in Head Injuries}

J. V. CRAwFord (London) discussed results obtained by two forms of treatment in a series of severe head injuries. First in a series of 12 cases showing a syndrome suggesting hypothalamic disturbance, A.C.T.H. was given. Two cases showed dramatic improvement, three cases improved enough to justify continuing and recovered. One case initially improved but relapsed and died. Six cases showed no effect, five of them died.

Secondly, 20 cases of severe head injury were subjected to chlorpromazine and cooling to $90^{\circ}$ to $92^{\circ} \mathrm{F}$. to try and assess any obvious benefit. They were classified as:

(a) Two cases of severe early concussive shock; treatment was not persisted with owing to hypotension, one died.

(b) Eleven cases in coma who were deteriorating; one patient rapidly improved and later recovered; three patients (also given A.C.T.H.) improved and 
eventually recovered; one patient with bouts of periodic respiration reverted rapidly to normal rhythm, slowly improved and recovered. Six patients (including two with A.C.T.H.) showed no real improvement-all died.

(c) Seven cases with hyperthermia (including five under $(b))$. All temperatures dropped below normaltwo died.

(d) Two cases with respiratory obstruction; one improved dramatically and recovered; the other showed insignificant improvement and died.

(e) Three cases of extreme restlessness; all responded to some degree-all recovered.

It was emphasized that except group (e) all cases were very ill and appeared to be deteriorating. It was fully realized that no conclusions could be drawn from so small a number.

\section{The Role of Acetylcholine in Head Injury}

BRodie Hughes (Birmingham) presented the results of some investigations into the role of acetylcholine in headinjured patients and discussed their significance. The presence and amount of acetylcholine in the C.S.F. was estimated by a bio-assay method using the isolated heart of Mya arenaria. In a control series of 200 neurological cases only occasional positive assays were encountered and the amounts recorded were quite small.

Forty-two assays were made in 20 head-injured patients and $43 \%$ of these were positive. Quantities varied, the highest recorded being 10 gamma \%. A correlation was then made between various physical factors in head injury and the presence and quantity of acetylcholine found. No close relationship could be demonstrated between acetylcholine levels and the state of consciousness, gross local damage to the brain, or generalized neuronal damage as estimated by the post-traumatic amnesia.

Statements by previous workers that the presence of acetylcholine was associated with low voltage "flat" E.E.G. records was not confirmed, most patients with positive assays having high voltage records with much delta activity. Return of the E.E.G. pattern towards normal rhythms and voltages did not appear to have any relationship to rise and fall of acetylcholine levels. Most commonly positive assays were obtained in the second or third week after injury. In a few cases early high levels were encountered and in one case there was a sharp terminal rise before death.

The effect of atropinization with doses as high as $1 / 10$ grain $(6.5 \mathrm{mg}$.) was tried but without effect in either positive or negative assay patients.

No clear conclusions were arrived at concerning the origin of the acetylcholine though the information available suggested that it originated from a specific group of cells. The late appearance of the substance suggested that the area of brain responsible was probably damaged by secondary changes after injury rather than by the primary injury. It was suggested that the finding of groups of chromophobic cells in the anterior hypothalamus in postmortem material from positive assay patients might provide a clue as to the site of injury responsible.

\section{Experimental Studies in Brain Swelling}

W. E. Stern (Los Angeles) reported the results of experimental laboratory techniques applied to the study of the phenomenon of cerebral swelling. Cerebral swelling was defined as a state of increased brain bulk with an attendant increase in C.S.F. pressure.

Intravenously administered distilled water $(40 \mathrm{ml} / \mathrm{kg}$. at $60 \mathrm{drops} / \mathrm{min}$. at $37^{\circ} \mathrm{C}$.) in 20 adult mongrel cats produced an elevation of cisternal C.S.F. pressure averaging $210 \mathrm{~mm}$. water above the starting pressure. Such changes were not noted when physiological salt solution was administered under similar circumstances. Brain water and electrolyte determinations were made by gravimetric and spectrophotometric analyses.

To elucidate the relationship between bulk increase of the swelling brain and C.S.F. pressure a second series of animals was studied whereby the brain was compressed by a balloon distended with known volumes of fluid. The compression was designed to produce the same C.S.F. pressure for the same duration of time as had occurred with the administration of distilled water alone. The intracranial volume was determined as well as, in a second series, the relationship between brain mass and intracranial volume. From data so accumulated it could be calculated that if water increase in the brain were the sole factor explaining the pressure increase it would require an increment of $2.5 \%$.

Although statistically significant increases in wate occurred in the grey matter of the brain of animas receiving distilled water and in animals previously pres pared with antidiuretic hormone followed by water, the increases were not of the calculated magnitude. Whe r electrolyte changes were recorded they were in the direction of sodium falls and potassium rises, especiall in the grey matter.

Brain compression by balloon methods and followe of by water administration produced maximum elevation of . cisternal pressure. The pressure elevation produced by water alone was not associated with the death of any animal. Brain compression techniques which produced the same pressure elevations were often associated with death of the animal. "Spontaneous" rises in C.S.F. pressure could be observed suggesting shifts and impaction of brain tissue. When compression was followed by water administration the death rate was maximal. Spontaneous drops in C.S.F. pressure occurred suggesting mechanisms related to but opposite in effect of the "triggering" mechanism which produced the spontaneous rises during active balloon compression.

Fluid shifts within, and to and from, brain tissue together with mechanical distortions and impactions or disimpactions probably determined the death or survival of the animals so studied.

\section{Ventriculo-peritoneostomy in Post-inflammatory Hydrocephalus}

J. MOORE ROBERTSON (Glasgow) reported on the treat- $\frac{7}{2}$ ment of 19 cases of post-inflammatory hydrocephalus of obstructive or communicating type by means of ventriculo- N peritoneostomy. Polythene tubing, used in early cases, was abandoned owing to its tendency to kink and break and was replaced by silicone tubing. It was stressed that 
silicone tubing had to be firmly secured owing to its tendency to slip.

Six cases of late hydrocephalus following apparent cure of tuberculous meningitis were treated. Age was from three to 12 years and the onset of symptoms 17 months to three years after treatment. Tuberculous infection recurred in only one case.

One case, a child of 5 years, died suddenly a year after operation. Death was thought to be due to tube blockage but this was not clearly established at necropsy. The remaining five cases had remained free from symptoms and signs of intracranial hypertension for periods of four months to three years since operation.

Five cases of acute hydrocephalus in the active stage of tuberculous meningitis were treated, aged from 11 months to 20 years. These patients showed progressive deterioration under treatment and developed hydrocephalus of obstructive or communicating type. One patient died 10 days after operation, two were progressing satisfactorily under treatment, and two had completed treatment and were well. The period of follow-up was from three months to two years.

Eight cases of hydrocephalus following septic meningitis were treated. In five of these polythene tubing was used. Four of these died of progressive hydrocephalus due to failure of the drainage procedure. The remaining case remained well for two years when the drainage tube broke in situ and symptoms recurred. The broken tube was replaced by a silicone drain with satisfactory results.

In the remaining three cases silicone tubing was used. One case died five months after operation from prolonged generalized sepsis which had started in neonatal life. Two patients were well four months and one year after operation.

An Unusual Cerebellar Tumour with Unusual Metastases ARNOLD DE VET (Wassenaar) reported the findings in the case of a young man, aged 21 years, who was operated on in July, 1953, for an intracerebellar tumour near the midline. It was a malignant growth, at that time considered to belong to the group of the medulloblastomata.

After subtotal surgical extirpation and a course of deep $x$-ray treatment, the patient had so far remained without symptoms of local recurrence. He developed, however, spinal metastases of which the first one, especially, was of a very unusual type.

Eighteen months after the cerebellar operation he began to show signs and symptoms of a spinal cord lesion at L.2, 3 level and four months later he was operated on again. A long, cylindrically-shaped, well-encapsulated tumour $(6 \times 1.5 \mathrm{~cm}$.) with a smooth surface and without any tendency to invade the surrounding structures was removed entirely and at the time of operation no one considered this neoplasm to be a metastasis of the cerebellar tumour. Microscopically, however, this was proved to be the case.

The patient, therefore, received a series of $x$-ray treatments to the spine. He recovered only partially and in the beginning of 1956 a total transverse lesion of the spinal cord at mid-thoracic (T.8) level developed.

The patient did not return for further treatment until August, 1956. Examination revealed the existence of a new spinal tumour. This time, however, the tumour was not so well encapsulated and there was diffuse infiltration of the leptomeninges. Nevertheless, this metastasis, the total length of which amounted to $12 \mathrm{~cm}$., was removed almost completely except for the thin layers of leptomeningeal infiltration; it also was of an unusual type.

Recovery was better than could be expected after a transverse lesion syndrome of such long standing. Another series of intensive $x$-ray treatments to the spine was given and the patient's condition remained fairly good.

The microscopical picture was the same in all three tumours. It was that of an extremely cellular neoplasm, with pleomorphous cells without protoplasm and very little stroma; there were numerous mitoses, the number of which varied considerably in different parts of the tumour. A strict classification of this glioma was not possible because of its marked anaplastic character.

\section{Parkinsonism Secondary to Cerebral Tumours}

A. Dickson Wright (London) reviewed reported instances of a Parkinson syndrome produced by cerebral tumours. It was noted that, whilst tumours located within the basal ganglia might be expected most often to produce the syndrome, the majority of reported cases were of tumours in the neighbourhood compressing and distorting these structures. Instances of the syndrome being produced by tumours in the parietal, parasagittal, and septum lucidum areas were noted.

Three personal cases in which Parkinsonism had been produced by sphenoidal wing meningiomas were reported; these appeared to be unique in the literature.

Case 1.-An adult female patient was noted to be developing a Parkinson syndrome and quickly deteriorated and had to abandon her employment. The true diagnosis was not suspected until she was admitted to hospital following a minor head injury. Examination revealed papilloedema and homonymous field loss. Ventriculography revealed the presence of a large subtemporal mass and at operation a huge sphenoidal wing meningioma was completely removed. This operation was followed by a remarkably rapid improvement and within a week the Parkinsonism had almost disappeared.

Case 2.-A 68-year-old woman had been suffering from dementia for a number of years. Finally admission to hospital became necessary and investigations, including angiography, demonstrated a large sub-temporal tumour. Very marked features of Parkinsonism were present on the right side only as well as right-sided pyramidal signs. The tumour was completely removed with rapid improvement in the Parkinsonism; there was only slight improvement in the dementia. Death occurred two and a half years later as a result of carbon monoxide poisoning. At necropsy there was no evidence of tumour recurrence.

Case 3.-A 60-year-old woman had suffered from headaches of a year's duration and had left her post as a secretary six months previously because of this pain. For eight months a tremor of the left hand and foot had been noticed and during this period a typical Parkinson syndrome developed. She had had a number of "giddy turns ", in one of which consciousness had been lost. She had also complained of frequency of micturition and occasional nocturnal incontinence. No abnormal physical signs were found other than those of Parkinsonism. Investigation by ventriculography and angiography revealed 
a large sphenoidal wing meningioma which was completely removed. The Parkinsonian features disappeared quickly after operation.

\section{Primary Tumours of the Thalamus}

K. W. E. PaIne (London) reported the findings in a series of 24 cases of primary tumour of the thalamus. Gliomata of the thalamus were found to account for $1 \%$ of all intracranial tumours and to occur in young adults of either sex.

Early obstruction of the third ventricle by the tumour resulted in the early onset of signs and symptoms of raised intracranial pressure and these were present in almost all of the patients by the time they sought treatment. Involvement of the internal capsule and optic radiation might result in hemiparesis, sensory loss, or hemianopia, and it was not unusual to encounter various forms of tremor in the involved limbs. This tremor might be a combination of weakness, sensory impairment, extrapyramidal dysfunction, or cerebellar incoordination. A group of symptoms which occurred commonly in patients with thalamic tumours was inequality of the pupils, poor pupillary reactions, and impairment of ocular movements, especially conjugate deviation. These signs were probably due to the spread of the tumour along the periaqueductal subependymal tissues of the brain-stem.

Mental changes were usually the result of increased intracranial pressure and hydrocephalus of the lateral ventricles and not of any specific effect of the tumour on the thalamus.

The ventriculographic appearances were described and these were quite characteristic. The pathology was not known in all cases but in view of the long survival of many patients the tumours were probably benign types of glioma.

The rational treatment in most instances seemed to be the relief of the third ventricle obstruction by means of a ventriculocisternostomy (Torkildsen's operation). Eleven of 24 patients were so treated with satisfactory results.

Stereotaxic Recordings from the Frontal and Temporal Lobes of Psychotics with and without Paroxysmal Episodes

S. L. SHERwOOD (London) reported on electroencephalographic recordings taken with the aid of a light weight stereotaxic instrument at 25 operations on 23 patients with behaviour disorders. Thirteen of these concerned the temporal lobes and 12 the frontal lobes. In each case records were taken throughout the lobe concerned through an array of nichrome-needle electrodes. Results were grouped in the following manner:

(1) Those with fits only and loss of consciousness in the fits; these patients showed paroxysmal activity or hyper-excitability of the brain tissue mainly in the cortex of the temporal lobe in question and some diffuse hyperexcitability throughout the lobe.

(2) Those with fits and affective disturbances with diminished insight; these showed similar phenomena but mainly confined to the inferior temporal cortex and the so-called rhinencephalic parts of the temporal lobe.

(3) Those with affective disorder and episodic disturbances of insight and consciousness; patients of this group showed hyper-excitability or paroxysmal activity in the large white fibre masses such as the internal capsule, corona radiata and external capsule, as well as parts of the temporal cortex.

(4) Those without seizures at present but a behaviour disorder of the dementia praecox type; these patients showed localized asynchronous paroxysmal activity where different areas reacted apparently independently.

(5) Those with purely affective disturbances; this group showed extensive synchronized and paroxysmal activity throughout the frontal lobes.

It was noted that the results of so small a series could not be used for generalization but so far the trends described appeared to be consistent.

\section{Sensory Neurone Selection in Relief of Pain}

R. M. MAHER (Rochdale) reported a simple and useful injection method for relief of pain in cancer. With the patient in the usual lateral position a spinal puncture was made above the pain-affected segments and phenol or silver nitrate in heavy conveying agents (" myodil" or glycerin) was injected. By a slight downward tilt of the patient the injection material was allowed to gravitate to lower segments.

At lumbar level $1 \mathrm{ml}$. of phenol 1 in 20 was used for injection. In resistant cases below mid-dorsal level $1 / 100$ grains $(0.65 \mathrm{mg}$.) silver nitrate per $\mathrm{ml}$. was added to the injection. For upper dorsal and cervical siteso $3.5 \mathrm{ml}$. phenol 1 in 20 was used. Radiographs following cervical injection showed a double line of " myodil " above and below, though the patient was lying on the 7 side. It was considered that this indicated a subdurak injection, the "myodil" having flowed round the cir? cumference of the narrow subdural space.

Using phenol 1 in 10 dilution it was thought that totab posterior root destruction at A fibre level was produced There was resultant cutaneous anaesthesia with bed sores and loss of proprioceptive sensation and a disability functionally equivalent to anterior root or cord destruction.

Using phenol 1 in 20 dilution produced selective destruction, probably at $\mathbf{C}$ fibre level, resulting in relief of pain with only minimal cutaneous analgesia and no sideeffects.

Results were reported in 97 cases. In seven cases phenol injections in both dilutions gave no relief. Necropsy showed in four cases that the spinal canal and meninges were heavily involved in growth.

In 51 cases immediate relief of pain was followed by relapse within five days. At necropsy in some of these cases the spine was invaded by growth and carcinoma cells demonstrated within the ganglia.

In 39 cases immediate and permanent effect was obtained.

It was suggested that for permanent relief of pain destruction of the ganglion cell was necessary. In the first group these cells may have been "sheltered" by carcinoma cells and in the second group "sheltering" may have also been present, the temporary effect being an effect on the nerve fibre alone. Extension of pain to distant segments or nerve groups and close proximity of primary growth to the spine both suggested direct 
invasion of ganglia by growth and results in these groups were poor. It was also considered that long duration of nerve involvement predisposed to sheltering of ganglion cells and results were worst in patients with long histories of neural involvement.

\section{Return to Russia}

SiR Geoffrey JefFerson (Manchester) gave an account of a visit to Russia as leader of a group of seven, selected by the British Medical Association and invited by the Academy of Medical Sciences and the Ministry of Health of the U.S.S.R.

It was reported that medical education had been separated from the universities for some $\mathbf{2 5}$ or more years and was conducted in special institutes. Since these were run on the same lines as universities with full professorial staffs the loss from separation was a small one. Some $40 \%$ of Russian surgeons were women and this held true mostly for neurosurgery, except at Kiev where out of a staff of 56 only two were women. Money for development and research seemed to be plentiful.

Visits were paid to the three principal neurosurgical institutes which were alone responsible for the training of neurosurgeons. They were the Institute Burdenko in Moscow (340 beds), Leningrad (200 beds), and Kiev (150 beds).

In Moscow the turnover of cases was some 3,000 a year, of which 800 were verified tumours. There were four or five main divisions, each with its own chief-tumours, injuries, infections, peripheral nerves, sympathetic surgery, and paediatric neurosurgery. Sub-division in this way was the general plan in all institutes. The Director, Professor Egarov, had two deputies, Professors Koreysha and Konyonski, and a staff of 60 , some 38 of whom would be allowed to operate independently.

In Leningrad (Director, Professor Lieut.-Gen. Shamov) there was a staff of 54 with Professor T. S. Vaskin in charge of tumour surgery and Dr. E. G. Loubenski of trauma. Dr. A. G. Zemckaya, one of the women surgeons, of whom there were 30 , pointed out an interesting genetic peculiarity of Russia in that anterior basal meningo-encephalocele was greatly more common than posterior. She had perfected a method of repair by closing the skull defect with plexiglass.

Professor Shamov, though Director, did little operative work at the Leningrad Institute, being chiefly engaged at the famous Military Academy.

In addition to the main institutes, in which all the postgraduate training was carried out, there were upwards of 26 other neurosurgical establishments spread over the U.S.S.R. as parts of general hospitals as far east as Vladivostock. These might have 10 to 60 beds and dealt mainly with urgent problems of injury and infection. They were established to deal with battle casualties and had been continued after the war in affiliation with the Leningrad Institute. For the more difficult cases a team would be dispatched from Leningrad or the case flown in.

The Kiev Institute was headed by an engaging and energetic young neurosurgeon, Professor A. I. Arutunov. He was interested in biochemical changes in lesions of the nervous system and had active laboratories working on these subjects.
In diagnosis reliance was placed on air pictures above everything and the small number of angiograms made were done by the neurosurgeons themselves. Aneurysms were seldom operated on, the cases remaining in the hands of physicians. Nor was much surgery carried out for epilepsy. The real concentration of effort was on brain tumours and trauma, though even in the tumour field intracapsular operations for acoustic neurinomas were the rule. Isotopes were being well used by Dr. Badmaev at the Leningrad Institute using $I^{131}$ and a scintillator scanner.

Everywhere research was very much tied to Pavlovian ideas which were used to explain clinical behaviour whenever possible. The most interesting physiological work was seen in Professor Sarkisov's Brain Institute in Moscow, a purely research unit with no patients. There they had inserted indwelling micro-electrodes into four or five cortical layers and correlated the animal's behaviour under conditioning or inhibition with the respective E.E.G. patterns. Professor Sarkisov had also just published a new and beautiful histological atlas of the brain.

They were everywhere received with great cordiality and no restrictions at all were placed on enquiries or visits. The Russian neurosurgeons claimed to be well acquainted with the western literature; journals and books were lavishly purchased and a good translation service was said to exist.

\section{On the Advantages of Opening Certain Intracranial Aneurysms}

J. R. GibBs (London) reported two cases of middle cerebral aneurysm and one of non-fistulous carotid aneurysm in the cavernous sinus as examples of the indications for opening aneurysms deliberately.

In middle cerebral aneurysms, the indications were (1) a large, dangerously thin sac, (2) a wide neck, and (3) an anatomical arrangement such as to endanger the patency of the parent vessel while the neck was being clipped or ligated. After temporarily occluding the middle cerebral artery, the aneurysm was opened, the collapsed sac held up by forceps and a clip or ligature placed accurately at the neck.

A guarding clamp was described for temporary proximal occlusion and it was recommended that such a clamp should be applied in all cases of middle cerebral aneurysm, whether opening was intended or not, before the dangerous dissection of the neck was attempted.

In the case of carotid aneurysm in the cavernous sinus, exposure and evacuation of the aneurysm with clipping of the internal carotid above it resulted in relief of severe facial pain after successive common and internal carotid ligations had proved unavailing.

\section{The Manipulative and Operative Treatment of} Backache and Sciatica

I. N. MACiver (Newcastle) presented a review of 3,000 cases of backache and sciatica. These had been treated during the past 15 years in Newcastle and comprised 708 cases treated conservatively, 1,538 cases treated by manipulation, and 1,056 cases treated by operation.

A review had been made in 1950 and disclosed that, although $74 \%$ returned to their original employment, 
$17 \%$ of these relapsed later, $20 \%$ returned to light work only, and $4 \%$ did not return to work at all. Overall $56 \%$ were considered satisfactory. Out of these $14 \%$ had backache, $23 \%$ backache and sciatica, and $7 \%$ leg pain only.

Survey in the unsatisfactory cases showed that they fell into the following groups.

(1) Those who had had no neurological signs or only minimal signs; in many of these no real lesion had been found at operation. A few had adherent roots, swollen red roots, enlargement of the root ganglion, or simple bony ridges.

(2) Those cases in which there was adhesion between a root and the disc. This usually occurred in cases with a long history and small fibrotic nodules in the annulus.

(3) Those cases which had ganglionitis or thickened adherent extradural tissues.

(4) Cases where permanent damage had occurred to roots or cord.

(5) Cases where a disc at a different level had been missed at operation.

Following this review policy with regard to treatment was changed. It was decided that only cases with gross neurological signs should be operated on. Early operation was considered essential and several spaces were explored if the exploration at the expected level was negative. Adherent roots were freed and "gelfoam" placed between the root and disc and those with ganglionitis or thickened extradural tissues had the posterior root divided.

From 1944 onwards all other cases were manipulated first. This was carried out under "pentothal" and "flaxidil". With the patient lying face downwards the spine was hyperextended. Thereafter they were encouraged to get about and active rehabilitation given. The object of this manoeuvre was to allow a nerve root, impacted on the summit of a disc prolapse, to slip to one or other side. In this group $90 \%$ were able to return to work. Of the remainder the majority were found to be elderly patients with mental depression. At the present time laminectomy was only carried out when manipulation had failed or the criteria mentioned before were present.

Since this régime had been adopted $70 \%$ of patients had returned to work and were considered satisfactory. The number of these complaining of leg pain had been considerably reduced to $6 \%$ but the number complaining of backache had remained the same. The problem of this residual backache was being investigated.

It was noted that disc lesions might follow normal stresses and strains if there was a congenital weakness or deficiency of the nutritive protein exudate. Four types of lesion were described. In the first practically the whole annulus was torn and the nucleus prolapsed into the rent. In the second the inner annulus fibres were partially torn with nucleus prolapse resulting in a much firmer swelling. In the third partial tearing of the outer fibres resulted in a firm greyish nodule to which the nerve root might become adherent; this type seemed to be particularly common in miners. In the fourth type the annulus became detached from its attachments to the vertebral bodies and displaced backwards.

\section{Denervation of Carotid Sinus in Carotid Sinus Epilepsy*}

S. Behrman and G. C. KNIGHT (London) said that in a proportion of cases loss of consciousness accompanying stimulation of the carotid sinus might be produced by a neural mechanism independent of any alteration in the cerebral blood flow, pulse rate, or blood pressure.

Two patients affected by this condition were described in whom loss of consciousness would occur suddenly without warning, often on change of posture, such as rotation of the chin or assumption of an upright position.

In both cases attacks could be provoked by compression of the carotid bifurcation on one side and both were completely relieved by excision of the tunica adventitia from the region of the affected carotid sinus.

Evidence was advanced to indicate that certain cases of Stokes Adams syndrome induced by carotid sinus stimulation embody a similar mechanism since loss of consciousness still occurred after atropinization and that either epilepsy or Stokes Adams seizures could be produced by stimulation applied elsewhere in the territory supplied by the glossopharangeal nerve. It would appear that the stimulation of certain receptors, which ordinarily give rise to cardio-inhibitory reaction, might instead engender epileptic activity.

Carotid sinus epilepsy would therefore appear to be one form of sensory precipitated epilepsy. Focal seizures might occur in certain cases.

\section{Neurofibromas of the Sacral Canal}

Stevens Dimant (Manchester) said that solitary neurofibromas of the sacral canal were rare. Three cases in the Manchester series of 92 spinal neurofibromas were reported. Analysis of other series showed an even smaller proportion of sacral neurofibromata. There is little reference in the literature to the characteristics of these tumours.

Case 1.-Mrs. L. B., aged 39, had for three years rightsided sciatica with recent spread of pain to the perineum and to the inside of the left thigh. On examination brown marks were present on the lower extremities. There was gluteal weakness with both lumbosacral sensory loss and an absent ankle jerk on the right side. The sacrum was tender and straight-leg raising limited. The cerebrospinal fluid contained $140 \mathrm{mg}$. \% of protein. Radiographs demonstrated sacral erosion and a myelographic block at L.5/S.1. At operation an intra- and extradural dumb-bell neurofibroma was found to have grossly expanded the sacral canal. Complete removal was possible.

Case 2.-Mrs. E. J., aged 60 , had suffered from pain between the buttocks and sciatica on the left side for five and a half years. Examination revealed hypoalgesia on the left buttock with absent ankle jerks. There was also sacral tenderness and limitation of straight-leg raising. In the cerebrospinal fluid $70 \mathrm{mg}$. \% of protein was found. Radiography showed destructive changes within the sacrum with foraminal widening and an arrest of " lipiodol " at L.5/S.1. At operation a large extradural

* Read at the Neurological Section, B.M.A. Annual Meeting, Brighton, 1956. Brit. med. J., 1956, 2, 1522. 
neurofibroma that had replaced much of the sacrum was exenterated.

Case 3.-Mr. W. J., aged 43, complained of lumbosacral backache nine years previously and sciatic pain on the left for the past two and a half years. An epidural injection of saline accentuated the pain and paralysed the left leg and sphincters. On examination, neurological signs implicated all the left sacral roots. The cerebrospinal fluid protein was normal. There was a myelographic block at L.5 and considerable erosion around the sacral canal. An extradural multicystic neurofibroma that had reduced the sacral walls to a shell was removed at operation.

The Manchester spinal neurofibromas showed an average duration of symptoms before operation of two years; the corresponding figure for sacral neurofibromas was six years. The large size of the sacral tumours also signified late diagnosis. Sciatic pain with signs of sacral root disease and local tenderness suggested an intrasacral tumour. The radiographic appearances of a lobulated expansion of the sacral canal with smooth, sclerosed walls and a lumbosacral myelographic block were distinctive of a neurofibroma of the sacral canal.

\section{The Effects of Section of the Pituitary Stalk in Malignant Disease}

Campbell Connolly (Birmingham) said that it had been shown by Russell (1956) ${ }^{1}$ that division of the pituitary stalk in man produced necrosis of the anterior lobe of the gland. Upon this finding in post-mortem cases and with a knowledge of the anatomy of the blood supply of the gland, she has advanced a theory that in the treatment of carcinoma of the breast section of the pituitary stalk at its junction with the gland and coagulation of its distal end might be as effective as total hypophysectomy.

Such a procedure would deprive the anterior lobe of its blood supply from the superior hypophysial arteries. But it was considered possible, provided that the postoperative condition remained satisfactory, that viability of a large part of the anterior lobe would be maintained by means of the inferior hypophysial arteries, as these vessels drained into the portal vessels within the substance of the gland.

In support of this view, two cases were described in which the stalk was divided and the gland removed 18 days and 10 days later. In one of these there was little necrosis of the anterior lobe and in the other approximately one quarter of the lobe remained viable. A third case was reported in which the stalk was divided and part of the gland was removed. At post-mortem examination, five and a half months later, complete regeneration of the anterior lobe was found.

It was concluded that, although division of the stalk might produce improvement in patients with hormonedependent carcinoma, such improvement was likely to be transient only as revascularization and regeneration of the anterior pituitary would take place. Hypophysectomy must therefore remain the operation of election.

\section{Pituitary Destruction by Injection of Radioactive Substances and Section of the Pituitary Stalk for Secondary Carcinoma}

F. L. Davies (London) reported an investigation into the value of colloidal radioactive chromic phosphate $\left(\mathrm{P}^{32}\right)$ for destroying the pituitary gland by local injection. The method had been used in animals (dogs and rats) and in patients suffering from secondary carcinoma.

As the results obtained by this method alone (five cases) were unsatisfactory, subsequent patients were treated by combined injection and stalk section (three cases) and with stalk sections alone (four cases). Two cases were excluded as they had only recently been operated upon. The operative technique had been described elsewhere. There had been no operative deaths.

The animal experiments confirmed the claims made by Rothenberg, Jaffe, Putnam, and Simkin $(1955)^{2}$ for radioactive chromic phosphate as a suitable substance for pituitary injection. It was found to be rapidly precipitated in its colloidal form on injection, and to have an intensely local destructive action while its dissemination in the body was negligible. It emitted $\beta$ rays only and had a convenient half-life of 14 days.

In the concentration used in the present series $(3-8 \mathrm{mc}$. in 0.2 to $0.4 \mathrm{ml}$.) it had not been possible to destroy the pituitary gland completely by injection alone. None of the patients showed clinical improvement. In one patient, however, " adrenal $17 \mathrm{OH}$ " excretion and thyroid function fell to a very low level in one month.

Injection of $\mathbf{P}^{32}$ plus stalk section and stalk section alone had produced marked and early depression of pituitary function. Four cases showed early subjective improvement.

Post-mortem examination of the pituitary region in three cases revealed maximum radioactivity in the pituitary fossa, there being a slight " scatter" superiorly and inferiorly. A very small amount was found in the liver, spleen, kidney, and thyroid gland.

Histologically, a variable amount of viable glandular tissue was present in the anterior part of the gland, destruction being greatest in one case of injection plus stalk section. A varying degree of damage to the hypothalamic region was noted in all three cases.

This work was continuing and it was hoped to overcome the present difficulty in detecting those cancers which were truly hormone dependent. Two tests were being assessed, the oestrogen test (Pearson) and the estimation of urinary mammotrophins. When selection was more certain it was hoped that the relatively simple procedure of stalk section with or without injection of radioactive substances would prove a satisfactory alternative to hypophysectomy.

\section{REFERENCES}

I Russell, D. S. (1956), Lancet, 1, 466.
2 Rothenberg, S. F., Jaffe, H. L., Putnam, T. J., and Simkin, B.
(1955). A.M.A. Arch. Neurol. Psychiat., 73, 193. 